\title{
Chemical Functionalization of Bacterial Cellulose Film for Enhancing Output Performance of Bio-Triboelectric Nanogenerator
}

\author{
Natcha Boonchai ${ }^{1}$, Wiyada Mongkolthanaruk ${ }^{2}(\mathbb{D})$, Viyada Harnchana ${ }^{1,3}(\mathbb{D})$, \\ Kajornsak Faungnawakij ${ }^{(1 D}$, Supree Pinitsoontorn 1,3,* (D) \\ 1 Materials Science and Nanotechnology Program, Department of Physics, Faculty of Science, Khon Kaen University, Khon \\ Kaen 40002, Thailand \\ 2 Department of Microbiology, Faculty of Science, Khon Kaen University, Khon Kaen 40002, Thailand \\ 3 Institute of Nanomaterials Research and Innovation for Energy (IN-RIE), NANOTEC-KKU RNN on Nanomaterials \\ Research and Innovation for Energy, Khon Kaen University, Khon Kaen 40002, Thailand \\ 4 National Nanotechnology Center, National Science and Technology Development Agency, 111 Thailand Science Park, \\ Pathum Thani, 12120, Thailand \\ * Correspondence: psupree@kku.ac.th (S.P.);
}

Scopus Author ID 6507816542

Received: 25.04.2021; Revised: 26.05.2021; Accepted: 30.05.2021; Published: 9.06.2021

\begin{abstract}
Triboelectric nanogenerator (TENG) is a promising technology for converting mechanical energy into electrical energy. In the present research, a bio-TENG based on bacterial cellulose (BC) was fabricated, and the performance was improved by surface modification. The BC films were chemically functionalized by phosphorylation and sulfonation processes. The FTIR technique confirmed the functional groups on the phosphorylated and sulfonated BCs. The hydrophobic/hydrophilic properties were studied and found that the unmodified $\mathrm{BC}$, as well as the functionalized $\mathrm{BC}$, were both hydrophilic. The structure and morphology of the $\mathrm{BC}$ nanofibers were investigated by SEM imaging. It was shown that after phosphorylation and sulfonation, the BC nanofiber surface became rougher, and the fibers were densely packed. The pores between the nanofibers almost disappeared. These have resulted from the coating of the phosphate and sulfonic functional groups on the $\mathrm{BC}$ nanofibers. For TENG measurement, the $\mathrm{BC}$ film was paired with PTFE under a single-electrode measuring mode. The functionalized BC showed improved output performance compared to the unmodified $\mathrm{BC}$, possibly due to the rougher and denser $\mathrm{BC}$ surface and the change in the $\mathrm{BC}$ triboelectric potential. This research demonstrated a novel but straightforward way to enhance the output performance of the bio-TENG.
\end{abstract}

Keywords: triboelectric nanogenerator; bacterial cellulose; nanocomposite; surface modification;

(C) 2021 by the authors. This article is an open-access article distributed under the terms and conditions of the Creative Commons Attribution (CC BY) license (https://creativecommons.org/licenses/by/4.0/).

\section{Introduction}

In recent years, triboelectric nanogenerator (TENG) has received much attention as a promising technology for converting waste mechanical energy into useful electrical energy [1, 2]. In principle, it is possible to harvest almost all types of mechanical energy by using TENGs $[3,4]$. Thus, TENG is a potential candidate as an energy source for portable electronic devices. TENG employs the energy conversion principle via the combination of electrostatic induction and triboelectric effect [5]. More recently, biocompatible, bioinspired, or biodegradable TENGs (bio-TENGs) have been developed using sustainable and eco-friendly biomaterials as 
their main constituents $[6,7]$. They have attracted interest from many research groups since they can be utilized for transient electronics, self-powered implantable, and wearable electronics [8]. For example, Kim et al. fabricated the TENG from silk fibroin nanofiber and showed the high voltage output over a large surface area [9]. A wide range of natural materials has been successfully incorporated to form the bio-TENGs, such as egg white [10], chitin [10], chitosan [11], potato starch [12], and other polysaccharides [13].

Cellulose is a very important natural polymer. It is abundant and cheap, making it an almost indispensable natural raw material for sustainable industrial development $[14,15]$. The cellulose-based TENGs have been continuously researched in recent years as a part of the bioTENGs for green and eco-friendly energy harvesting systems. For example, cellulose nanofibril (CNF) hydrogel prepared from the wood pulp was attached to the indium tin oxide (ITO)/polyethylene terephthalate (PET) substrate as a positive electrode for TENGs. When pairing with fluorinated ethylene propylene (FEP) film as a negative electrode, the open-circuit voltage ( $\mathrm{V}_{\mathrm{oc}}$ ) and short-circuit current ( $\mathrm{I}_{\mathrm{sc}}$ ) of $\sim 5 \mathrm{~V}$ and $\sim 7 \mu \mathrm{A}$, were obtained [16]. In another work, $\mathrm{CNF}$ was impregnated into the porous structure of the cellulose microfiber (CMF) skeleton. The nanostructured cellulose-based paper was used as a triboelectric layer in the bioTENG used for self-powered healthcare products [17]. Furthermore, the bio-TENG was fabricated from highly porous CNF aerogel [18]. The porous structure improved the contact area and the electrostatic induction, which resulted in the enhanced TENG outputs.

Apart from natural cellulose from plants, bacterial cellulose (BC) has been used as an electrode for bio-TENGs. BC is grown by culturing bacteria strains under the right condition. It exhibits several unique characteristics, such as a three-dimensional nanofiber network structure, a high degree of polymerization, good mechanical properties, and biocompatibility [19-21]. An example of the BC-based TENGs was reported by Kim et al. [22], which dissolved BC hydrogel in ethyl acetate solution before regenerating it on a copper foil. The TENG output power was $4.8 \mathrm{~mW} / \mathrm{m}^{2}$ at a matched load resistance [22]. However, the method in preparing the $\mathrm{BC}$ film in this work involved the solubilization of the $\mathrm{BC}$ hydrogel. The major drawback of this process is that the unique structure of $\mathrm{BC}$, the porous network of three-dimensional nanofiber, was destroyed, and the crystallinity of $\mathrm{BC}$ also changed from cellulose I to cellulose II [22]. Additionally, the regenerated BC films were attached to electrodes using adhesive tapes $[12,23]$, creating an additional interface that reduced electron transfers across the interfaces. Alternatively, Jakmuangpak et al. fabricated the BC-TENGs by gradually drying BC hydrogel on a conductive substrate [24]. The BC film was adhesively bonded directly with the substrate without any use of adhesive tapes. This method directly bonded BC with the substrate and preserved the three-dimensional nanofiber network structure of $\mathrm{BC}$.

In the present paper, we followed the fabrication method proposed by Jakmuangpak et al. [24], but we further enhanced the TENG output performance by functionalizing the surface of BC. The chemical functionalization of $\mathrm{BC}$ has been proved to enhance the output voltage of TENGs. Yao et al. reported the fabrication of bio-TENGs made of CNF fibers and chemically functionalized with nitro and methyl groups [25]. Upon contact, the nitro-CNF, with excellent electron-withdrawing property, acquired a negative charge, whereas the positive charge was induced in the methyl-CNF due to an electron-donating functionality. As a result, the output voltage was enhanced due to the change in the tribopolarities of functionalized CNF. Therefore, in this work, we performed surface functionalization of $\mathrm{BC}$ using two processes: phosphorylation and sulfonation. Both processes are well known for significantly altering the surface properties of the original cellulose, such as surface hydrophobicity or dispersibility in 
specific solvents [26]. We found the enhancement of the output performance of our BC-TENGs after treatments. The chemical functional groups, morphology, and properties as well as the TENG performance were analyzed and discussed.

\section{Materials and Methods}

\subsection{BC cultivation.}

The process for cultivating $\mathrm{BC}$ was adapted from [24]. The bacterial strain Komagataiebacter nataicola (strain TISTR 975) was cultivated in a medium consisting of 100 $\mathrm{g}$ of anhydrous D-glucose (Ajax Finechem) and $10 \mathrm{~g}$ of yeast extract powder (HiMedia) in $1 \mathrm{~L}$ of de-ionized (DI) water. After cultivation at $30^{\circ} \mathrm{C}$ for $24 \mathrm{~h}$ in a shaker incubator, $1 \mathrm{~mL}$ of the cell suspension was transferred into a new container of a fresh liquid culture medium. The static cultivation at $30^{\circ} \mathrm{C}$ was then performed for 3 days. After that, the BC pellicle with $2 \mathrm{~mm}$ thick and $70 \mathrm{~mm}$ wide, was harvested. It was purified by boiling in DI water and soaking in $\mathrm{NaOH}$ solution. Then, $\mathrm{pH}$ was neutralized by rinsing with DI water several times. The purified $\mathrm{BC}$ hydrogel was kept in a refrigerator $\left(<4^{\circ} \mathrm{C}\right)$ before further use.

\subsection{Surface functionalization.}

For the phosphorylation of $\mathrm{BC}$ [27], $80 \mathrm{~g}$ of urea was mixed with $200 \mathrm{~cm}^{3}$ dimethylformamide (DMF) solution. The BC hydrogel was immersed in the prepared solution for $1 \mathrm{~h}$ with continuous stirring. Then, the solution was heated to 70,100 , or $130{ }^{\circ} \mathrm{C}$, and $40 \mathrm{~g}$ of phosphoric acid $\left(\mathrm{H}_{3} \mathrm{PO}_{4}\right)(80 \% \mathrm{v} / \mathrm{v})$ was gradually dropped into the solution. The reaction was continued for $4 \mathrm{~h}$. After cooling down to room temperature, the BC hydrogel was washed with the diluted 1-propanol solution, following by the $0.1 \mathrm{M}$ hydrochloric $(\mathrm{HCl})$ solution. It was finally rinsed in DI water several times until the $\mathrm{pH}$ of 7 was reached and kept in DI water before further use.

The sulfonation of $\mathrm{BC}[28]$ was done as follows. The $\mathrm{BC}$ hydrogel was immersed in the sodium periodate $\left(\mathrm{NaIO}_{4}\right)$ solution $(0.02 \mathrm{M}$ and $0.04 \mathrm{M})$ with gentle stirring at room temperature. After 24 and $48 \mathrm{~h}$, it was removed from the solution and rinsed with DI water. Then, it was further soaked in $5 \mathrm{wt}$.\% sodium bisulfate $\left(\mathrm{NaHSO}_{3}\right)$ solution at $50{ }^{\circ} \mathrm{C}$ for $5 \mathrm{~h}$. Finally, the BC hydrogel was cleaned with DI water several times until the $\mathrm{pH}$ of 7 was reached.

The chemical functionalizations of BC are schematically illustrated, as shown in Fig 1.

\subsection{Characterization.}

Before subjecting to any characterization techniques, the $\mathrm{BC}$ hydrogel was freeze-dried to obtain the $\mathrm{BC}$ aerogel to preserve its structure. The crystalline structure of the pure $\mathrm{BC}$ sample was examined using X-ray diffraction (XRD) with a diffractometer employing $\mathrm{Cu}-\mathrm{K} \alpha$ radiation (PANalytical, Empyrean, USA). The functional groups of the pure BC and treated BC sheets were investigated using Fourier transform infrared (FTIR) spectroscopy (Bruker, TENSOR27, Germany) within a wavenumber range of 4000 to $600 \mathrm{~cm}^{-1}$. A field emission scanning electron microscope (FESEM) (FEI, Helios, USA) was used to observe the surface morphology of the samples. The samples were gold-coated before imaging with the SEM to improve conductivity. To test the hydrophilicity/hydrophobicity of the samples before and after chemical functionalization, the samples were subjected to the water contact angle (WCA) measurement using a specialized instrument (FTA1000 Drop Shape Analysis System, UK). 

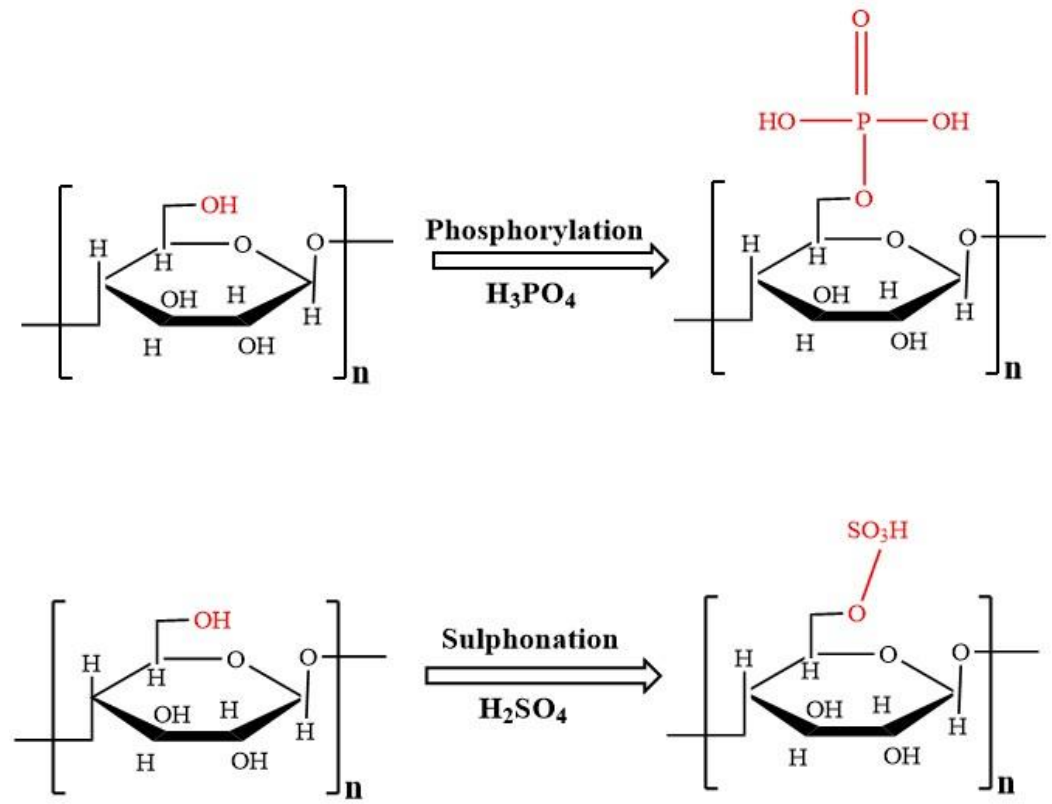

Figure 1. The schematic illustration of the phosphorylation and sulfonation of BC.

\subsection{Fabrication of BC-TENG and TENG measurement.}

To make the electrode for TENG measurement, the pure $\mathrm{BC}$ hydrogel, the phosphorylated $\mathrm{BC}$ hydrogel, or the sulfonated $\mathrm{BC}$ hydrogel was placed on a conductive glass substrate made from indium tin oxide (ITO). The hydrogel was allowed to gradually dried on the substrate. To speed up the process, it was put in an oven set at $60{ }^{\circ} \mathrm{C}$. Once dried, the flat thin BC film was formed on the ITO glass with very strong bonding. The TENG measurement was tested under a vertical contact-separation mode with a single-electrode [29]. The BC film and a Teflon plate were used as friction materials in the lower and upper parts. The substrate of the BC film (the ITO glass) in the lower section was only connected to the ground. An oscilloscope (RIGOL DS1054) and a digital multimeter (KEITHLEY DMM6500) were used for the voltage and current output measurement. The TENG voltage and current outputs were measured at room temperature with $35-40 \%$ humidity.

\section{Results and Discussion}

\subsection{XRD analysis.}

The XRD pattern of the untreated BC is shown in Fig. 2. The characteristic peaks of $\mathrm{BC}$ are observed at $2 \theta$ of $14.8^{\circ}, 17.2^{\circ}$ and $23.1^{\circ}$ corresponding to the Miller indices of $(1 \overline{1} 0)$ (110) and (200), respectively [30, 31]. No other peaks can be found indicating that the biosynthesized $\mathrm{BC}$ is in its single-phase without any impurity. The XRD of the phosphorylated $\mathrm{BC}$ and the sulfonated $\mathrm{BC}$ did not show any difference from the pure $\mathrm{BC}$, which is understandable since the XRD only examined the crystal structure but not the surface functionality. It indicates that phosphorylation and sulfonation do not change the $\mathrm{BC}$ crystal structure. 


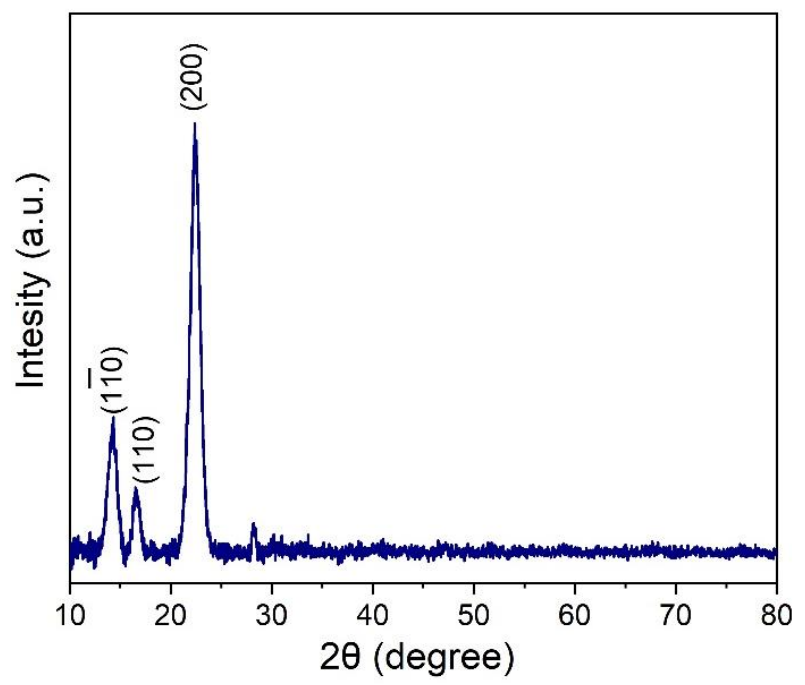

Figure 2. The XRD pattern of the oven-dried BC sheet.

\subsection{FTIR analysis.}

The FTIR spectra of the pure $\mathrm{BC}$ and the phosphorylated $\mathrm{BC}(\mathrm{PBC})$ are shown in Fig. 3. The samples PBC-70, PBC-100, and PBC-130 represent the phosphorylated BC (PBC) treated at $70{ }^{\circ} \mathrm{C}, 100{ }^{\circ} \mathrm{C}$ and $130{ }^{\circ} \mathrm{C}$, respectively. The broadband is observed at the wavenumber of $3600-3000 \mathrm{~cm}^{-1}$ which is assigned to the stretching of the hydroxyl group ($\mathrm{OH})$ of the glucose molecules [31]. This band is partially overlapped with the asymmetrical stretching of the methyl group (-CH) at the wavenumber of $2900-2800 \mathrm{~cm}^{-3}$ [32]. After phosphorylation, the band of the hydroxyl group stretching is reduced, which is due to the reaction with phosphoric acid $\left(\mathrm{H}_{3} \mathrm{PO}_{4}\right)$, resulting in new bands at $1230 \mathrm{~cm}^{-1}, 900-940 \mathrm{~cm}^{-1}$ and $834 \mathrm{~cm}^{-1}$, which are corresponding to $\mathrm{P}=\mathrm{O}, \mathrm{P}-\mathrm{OH}$, and $\mathrm{P}-\mathrm{O}-\mathrm{C}$ vibrations, respectively [27], indicating the formation of the phosphate group. However, these bands may be difficult to observe because they are in the same range as the $\mathrm{C}=\mathrm{O}$ and $\mathrm{C}-\mathrm{H}$ bands of the pristine $\mathrm{BC}$.

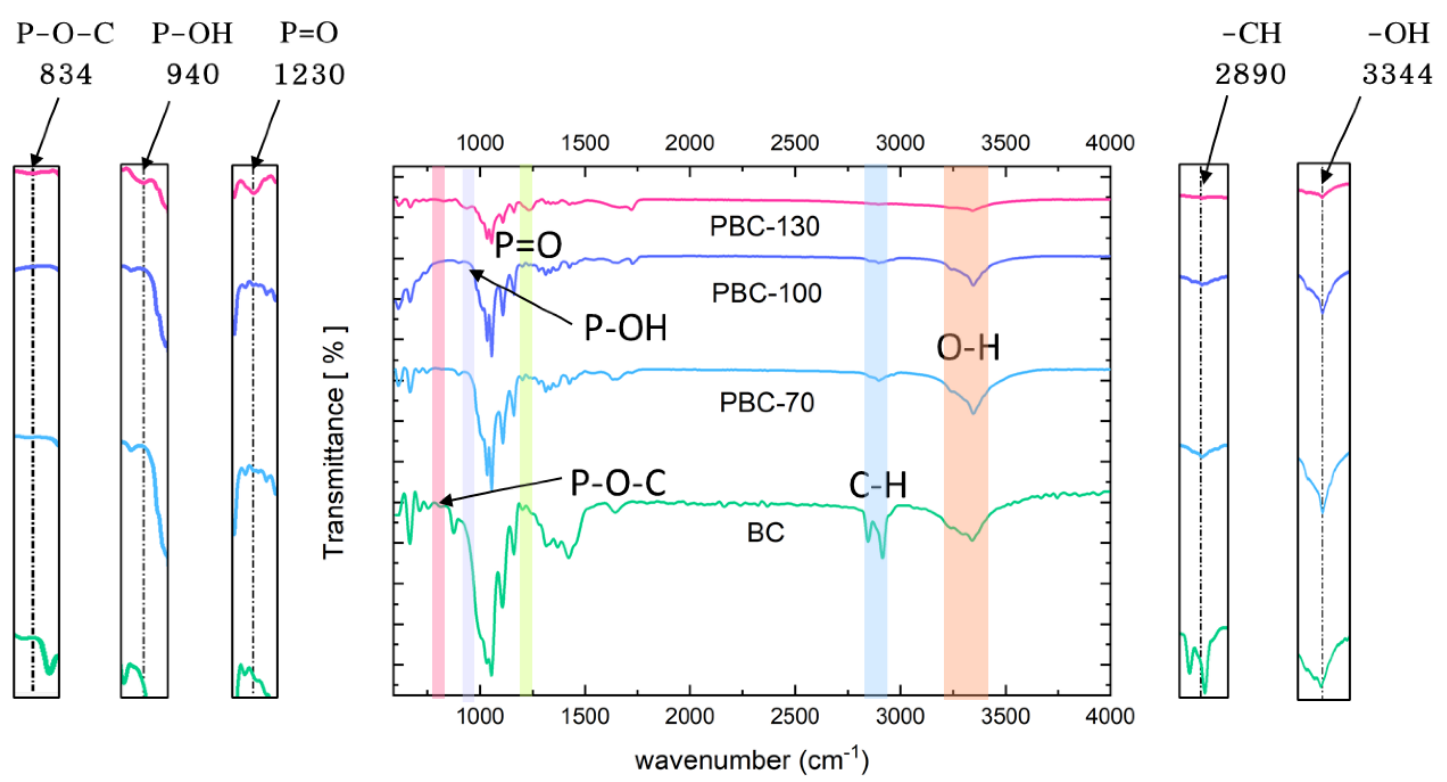

Figure 3. The FTIR spectra of the $\mathrm{BC}$ and the phosphorylated $\mathrm{BC}(\mathrm{PBC})$ treated at $70{ }^{\circ} \mathrm{C}(\mathrm{PBC}-130), 100{ }^{\circ} \mathrm{C}$ (PBC-100), and $130{ }^{\circ} \mathrm{C}(\mathrm{PBC}-130)$.

For the sulfonated BC (SBC), the FTIR spectra are shown in Fig. 4. The samples were immersed in $\mathrm{NaIO}_{4}$ solution for 24 or $48 \mathrm{~h}$, with a concentration of $0.02 \mathrm{M}$ or $0.04 \mathrm{M}$. Like 
phosphorylation, the sulfonated $\mathrm{BC}$ showed the $-\mathrm{OH}$ stretching reduction since it is bonded with the sulfonic acid group. The new band evidence this at $1360 \mathrm{~cm}^{-1}, 1062 \mathrm{~cm}^{-1}$, and $613 \mathrm{~cm}^{-}$ ${ }^{1}$ associated with the $\mathrm{S}=\mathrm{O}$ and $\mathrm{S}-\mathrm{O}$ vibrations of the sulfonic group [28]. However, these bands also overlap with the existing band of the pure $\mathrm{BC}$, such as the $\mathrm{C}-\mathrm{O}$ vibration at $1062 \mathrm{~cm}^{-1}$.

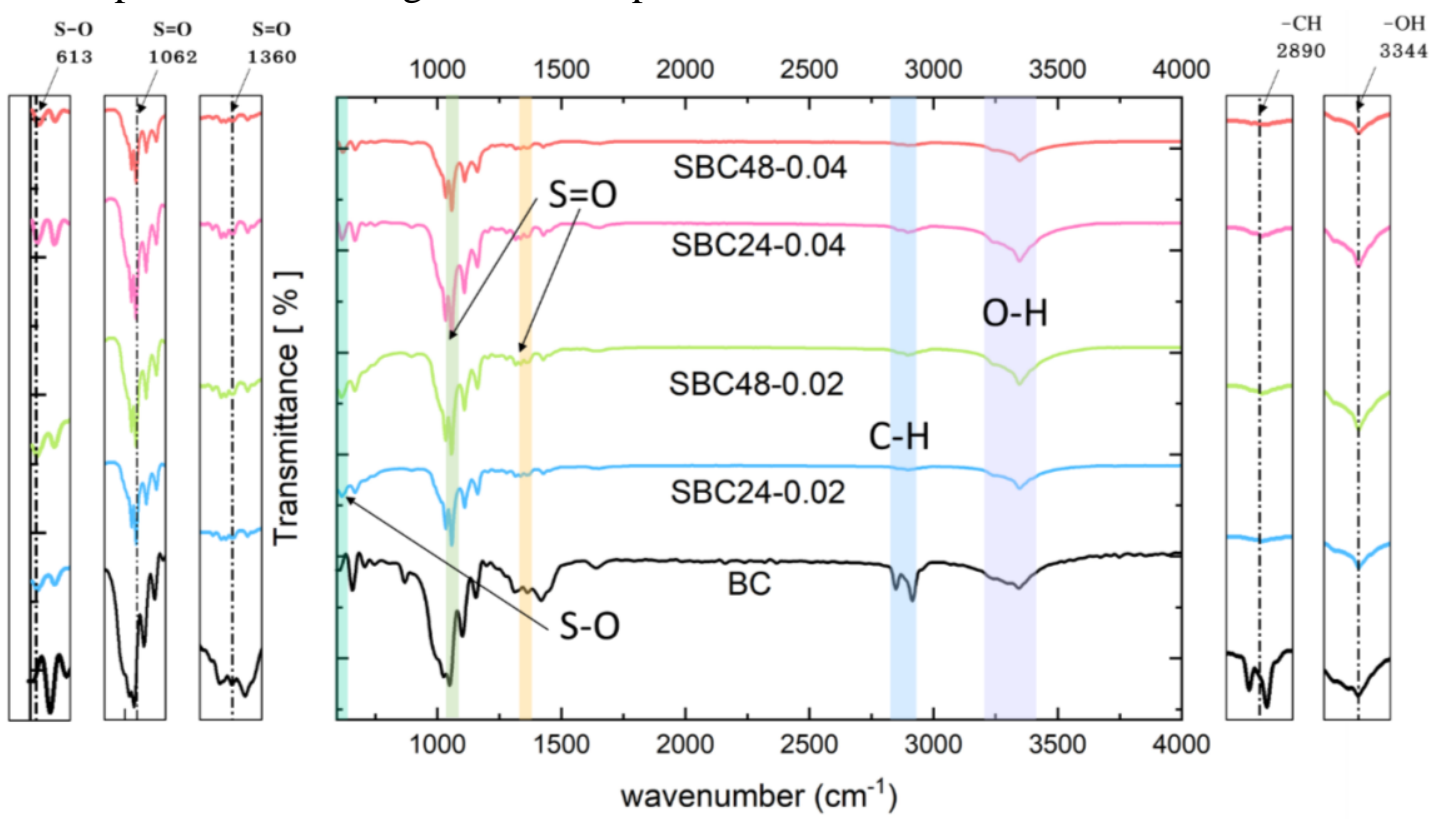

Figure 4. The FTIR spectra of the BC and the sulfonated BC (SBC) immersed in $\mathrm{NaIO}_{4}$ solution for 24 and $48 \mathrm{~h}$ with the concentration of $0.02 \mathrm{M}$ and $0.04 \mathrm{M}$.

\subsection{Water contact angle measurement.}

To determine hydrophobic/hydrophilic properties of the $\mathrm{BC}$ and the surface modified $\mathrm{BC}$, the $\mathrm{BC}$ membranes were subjected to the water contact angle measurement as shown in Fig. 5 and Fig. 6 for the phosphorylated BC (PBC) and the sulfonated BC (SBC), respectively.

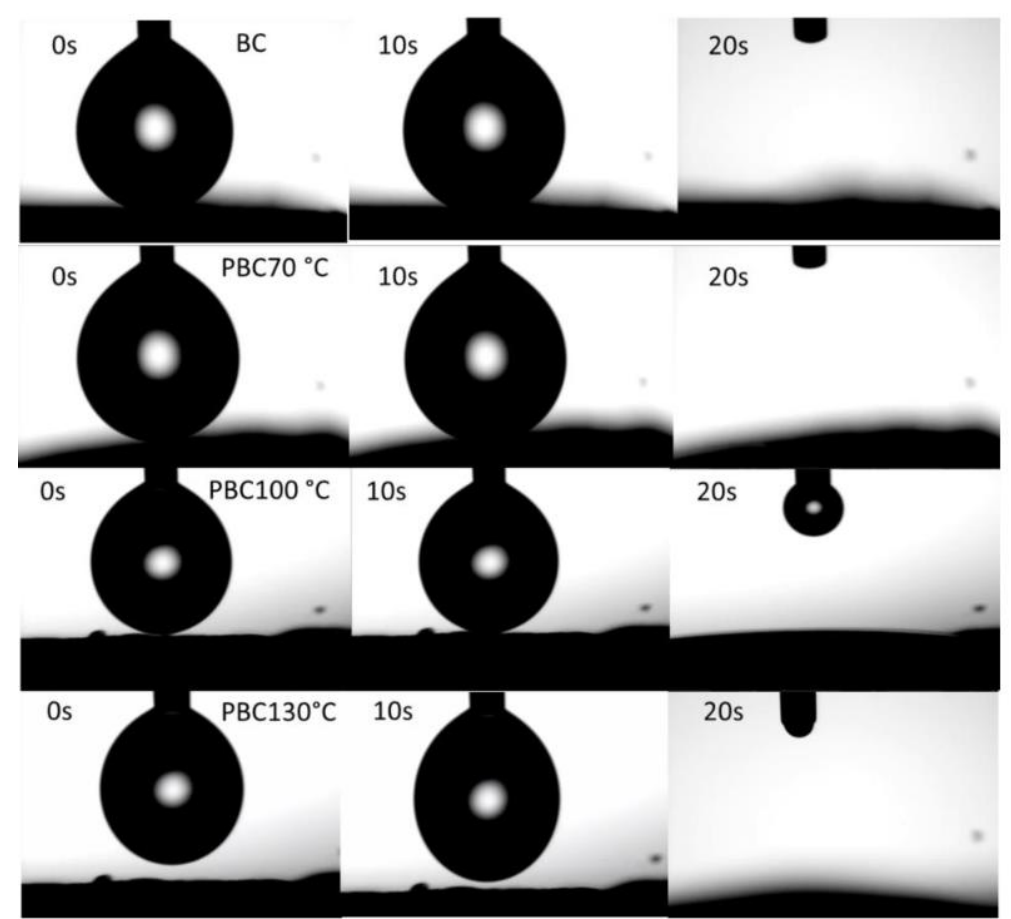

Figure 5. The water contact angle measurement of the $\mathrm{BC}$ and the phosphorylated $\mathrm{BC}(\mathrm{PBC})$ treated at $70{ }^{\circ} \mathrm{C}$ (PBC-130), $100{ }^{\circ} \mathrm{C}$ (PBC-100) and $130{ }^{\circ} \mathrm{C}$ (PBC-130). 
The pure BC shows the hydrophilic properties as expected since many hydroxyl groups are on the cellulose chains. As soon as a water droplet was dropped on the BC surface, it was absorbed completely. A similar observation was found in the case of the PBC and SBC surfaces, indicating the hydrophilic properties of both PBC and SBC. Altering the phosphorylation conditions, i.e., changing heat treatment temperature in the phosphorylation process, did not affect the hydrophilicity.

Similarly, changing the sulfonation concentration did not show any effect on the hydrophilic properties. The water droplet was absorbed almost immediately upon contact with the surface. The reasons for the hydrophilic properties of the PBC and SBC samples are due to the presence of phosphate and the sulfonic groups on the cellulose chains. These groups are polar functional groups, making electrostatic interaction with water molecules, resulting in hydrophilic properties.

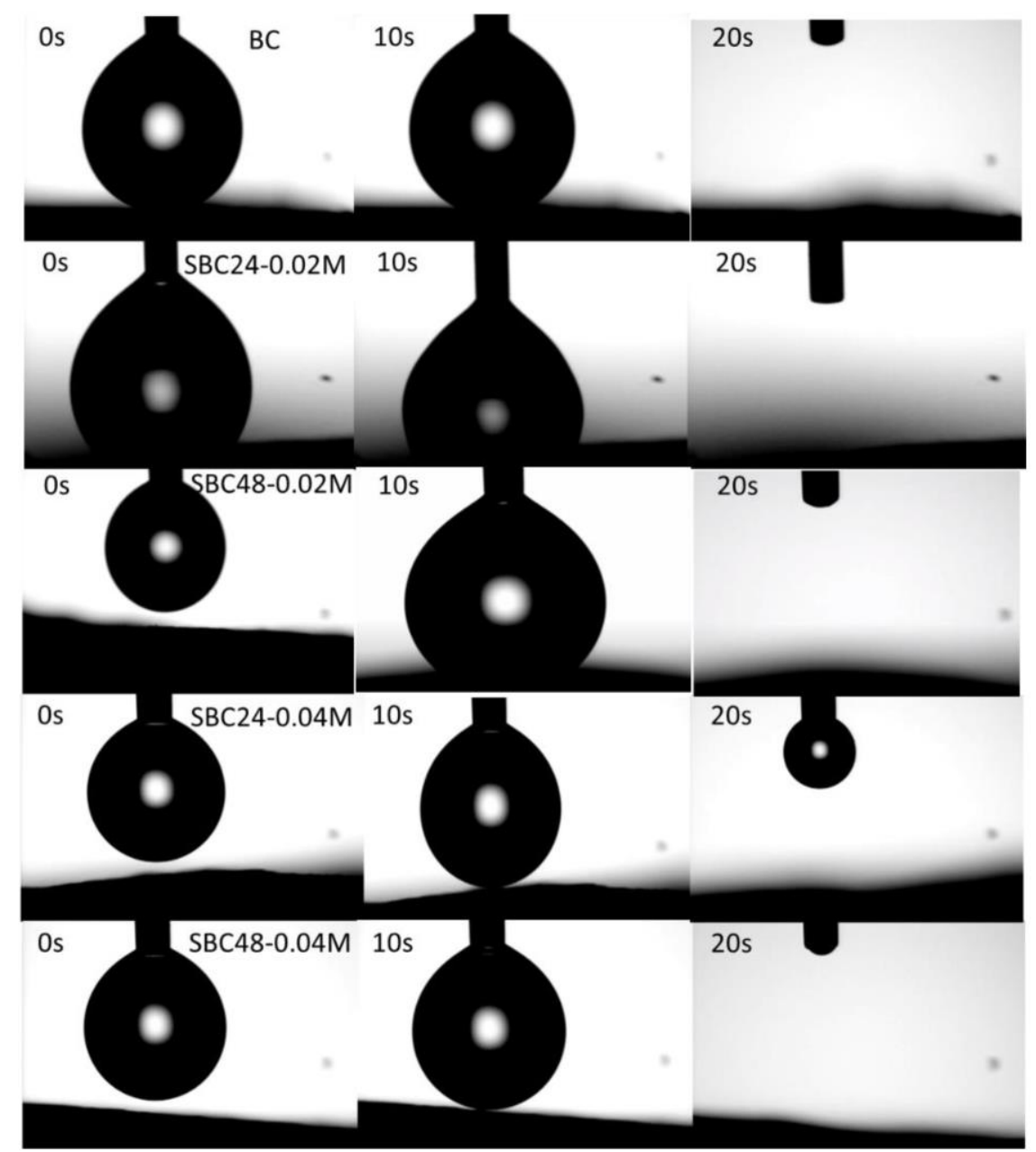

Figure 6. The water contact angle measurement of the $\mathrm{BC}$ and the sulfonated $\mathrm{BC}$ (SBC) immersed in $\mathrm{NaIO}_{4}$ solution for 24 and $48 \mathrm{~h}$ with the concentration of $0.02 \mathrm{M}$ and $0.04 \mathrm{M}$.

\subsection{SEM images.}

The surface morphology of the samples was investigated by using SEM imaging. Fig. 7 shows the SEM micrographs of the BC and phosphorylated BC (PBC). The surface of the pure $\mathrm{BC}$ consists of the interwoven nanofibers in a three-dimensional structure. High porosity can be observed. The average nanofiber diameter is about $50 \mathrm{~nm}$. When the $\mathrm{BC}$ was treated by phosphorylation, the phosphate functional groups were coated on the fiber surface. This is evidenced by the larger nanofiber diameters of the phosphorylated samples. Increasing the 
treatment temperature led to the larger fiber sizes of $110 \mathrm{~nm}, 120 \mathrm{~nm}$, and $150 \mathrm{~nm}$ for PBC-70, PBC-100, PBC-130, respectively. Moreover, the nanofibers were densely packed together with the obvious decrease of the porosity after phosphorylation. Particularly, for PBC-130, the nanofibers were so densely packed that the porous structure between the nanofibers was completely filled. This is due to the relatively high temperature at $130{ }^{\circ} \mathrm{C}$, which can kinetically drive the phosphorylation process, resulting in a thick coating of the phosphate groups on the BC nanofibers.

The SEM images of the BC and the sulfonated BC (SBC) are illustrated in Fig. 8. Similar to the PBC, the SBC shows the increase in the fiber diameters and the decrease in porosity after sulfonation compared to the pure $\mathrm{BC}$. The average fiber diameter increased from $50 \mathrm{~nm}$ for the pure BC to $70 \mathrm{~nm}, 80 \mathrm{~nm}, 90 \mathrm{~nm}$, and $90 \mathrm{~nm}$ for SBC24-0.02, SBC48-0.02, SBC24-0.04, and SBC48-0.04, respectively. This is due to the coating of the sulfonic group on the surface of the cellulose nanofibers. The changes in the surface morphology have an influential effect on the TENG output performance, as described in the next section.
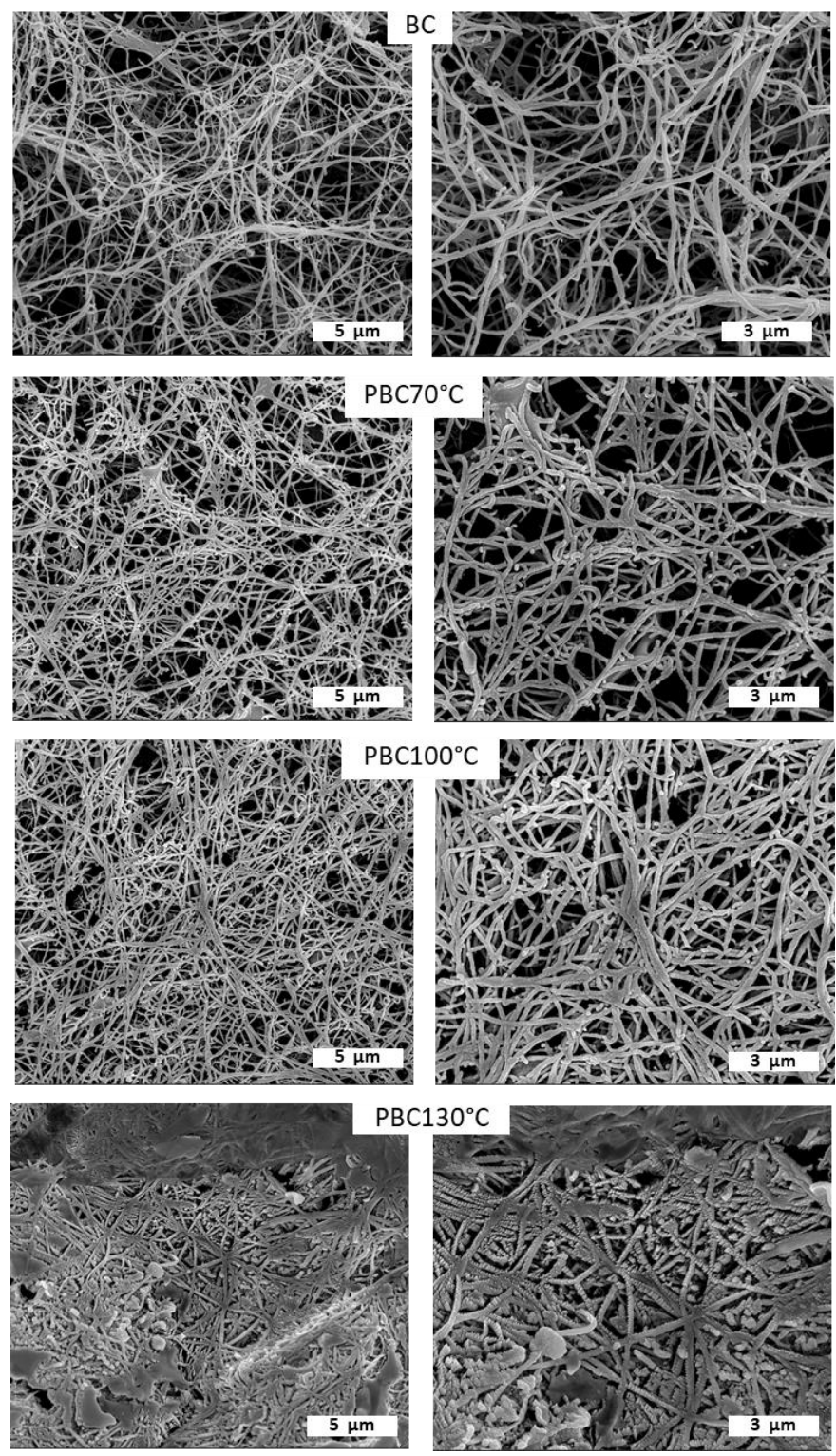

Figure 7. The SEM micrographs of the $\mathrm{BC}$ and the phosphorylated $\mathrm{BC}(\mathrm{PBC})$ treated at $70{ }^{\circ} \mathrm{C}(\mathrm{PBC}-130), 100$ ${ }^{\circ} \mathrm{C}$ (PBC-100), and $130{ }^{\circ} \mathrm{C}$ (PBC-130). Magnification: 15,000x (lef) and 30,000 (right). 

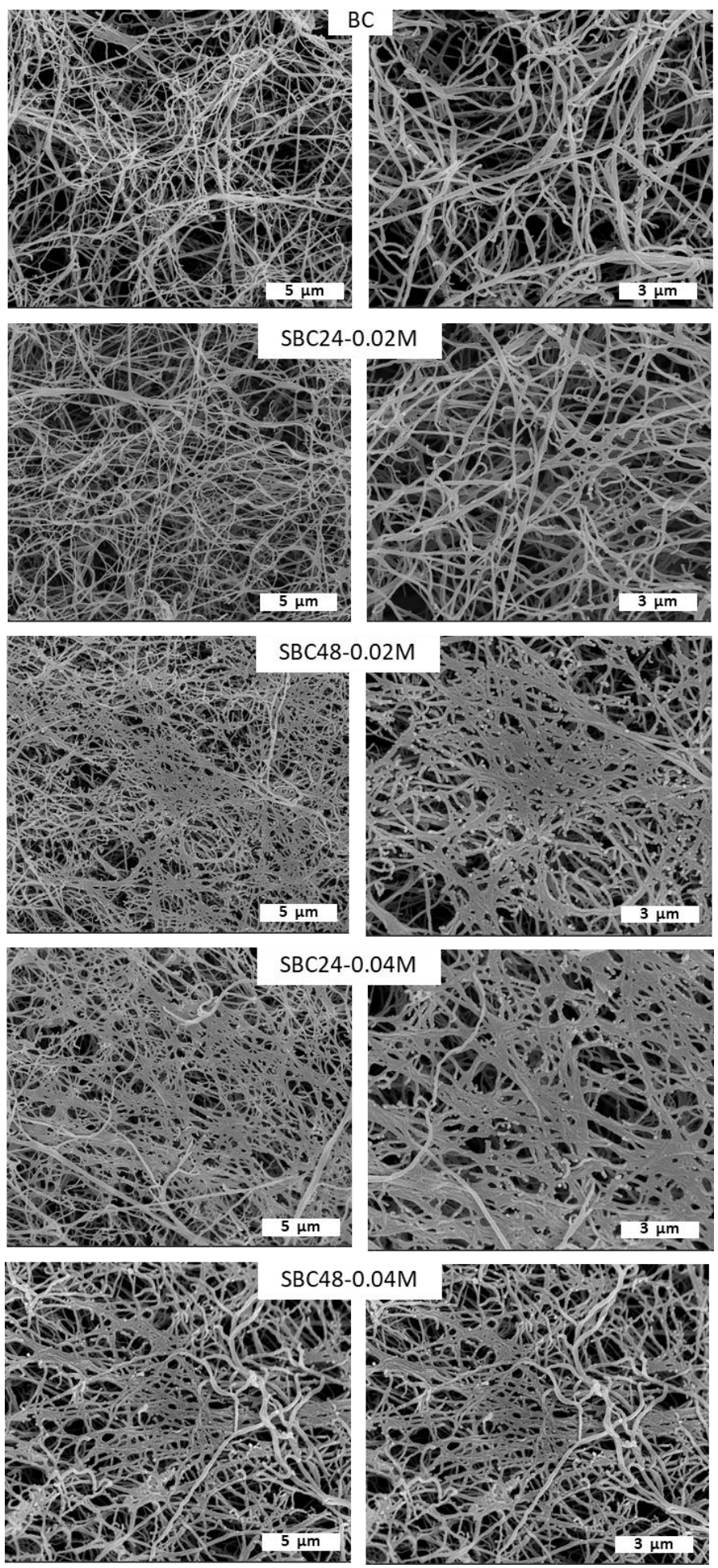

Figure 8. The SEM micrographs of the $\mathrm{BC}$ and the sulfonated $\mathrm{BC}$ (SBC) immersed in $\mathrm{NaIO}_{4}$ solution for 24 and $48 \mathrm{~h}$ with the concentration of $0.02 \mathrm{M}$ and $0.04 \mathrm{M}$.

\subsection{TENG output performance.}

After fabrication of PBC and SBC as the friction layer for TENG, the output signals were measured, and the results are shown in Fig. 9. The voltage and current outputs of the pure $\mathrm{BC}$ were $54.4 \mathrm{~V}$ and $4.96 \mu \mathrm{A}$. These values are comparable to other previous reports [22, 24]. After surface modification, the TENG output performances are clearly changed. For the phosphorylated samples, the output voltages were $48.8 \mathrm{~V}, 72.8 \mathrm{~V}$, and $76.7 \mathrm{~V}$ for PBC-70, PBC-100, and PBC-130, respectively, whereas the output currents were $4.32 \mu \mathrm{A}, 5.90 \mu \mathrm{A}$, and 
$6.35 \mu \mathrm{A}$, for PBC-70, PBC-100, and PBC-130, respectively. There is a trend that increasing the phosphorylation temperature led to the improved TENG performance. Similarly, the sulfonated samples also show the improvement of the TENG performance. From Fig. 9, the output voltages of the SBC were found to be $50.4 \mathrm{~V}, 55.2 \mathrm{~V}, 59.4 \mathrm{~V}$, and $64 \mathrm{~V}$ for SBC240.02, SBC48-0.02, SBC24-0.04, and SBC48-0.04, respectively. The output current was also increased with sulfonation with the values of $4.44 \mu \mathrm{A}, 4.49 \mu \mathrm{A}, 5.56 \mu \mathrm{A}, 5.94 \mu \mathrm{A}$ for SBC240.02, SBC48-0.02, SBC24-0.04, and SBC48-0.04, respectively. Thus, it can be concluded that the surface modification of $\mathrm{BC}$ by either phosphorylation or sulfonation can improve the TENG performance (both voltage and current outputs) in comparison to the unmodified BC.

The output performance of the TENG depends on several parameters, such as the pair of friction materials, frequency, forces, mode of contacts, environments, etc. However, if all other parameters are controlled, and only the friction materials are changed, the enhanced voltage and current output can be attributed to a few factors. Firstly, surface roughness can play a crucial role.

(a)

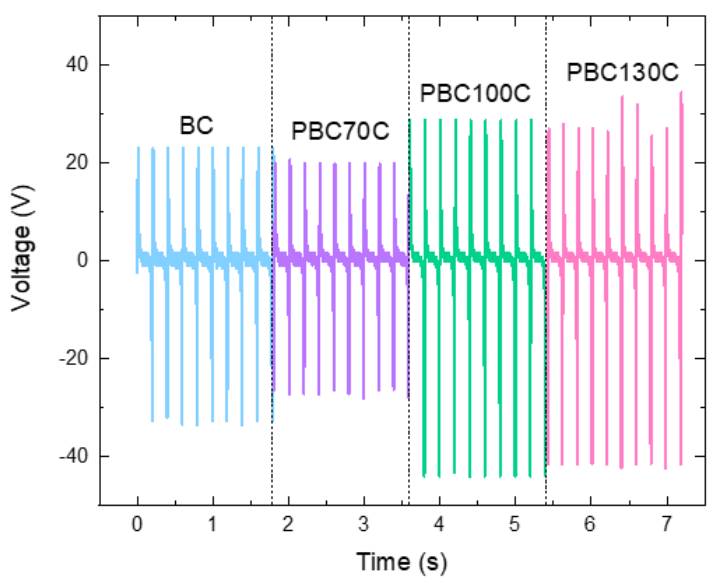

(c)

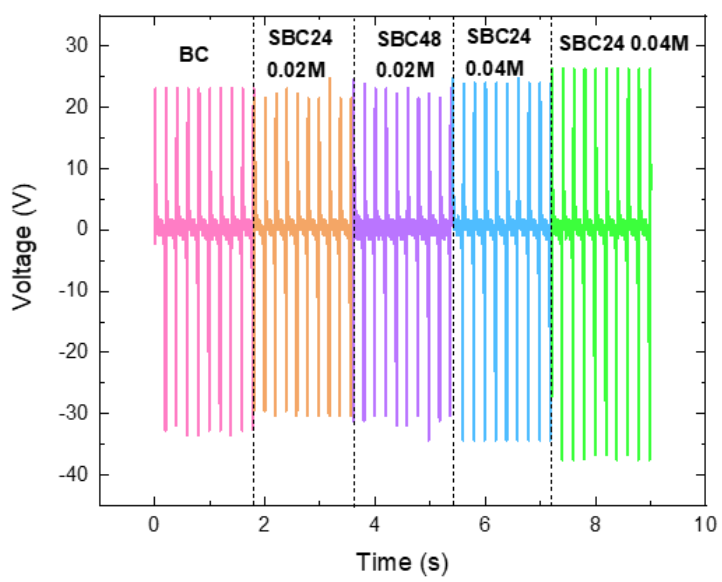

(b)

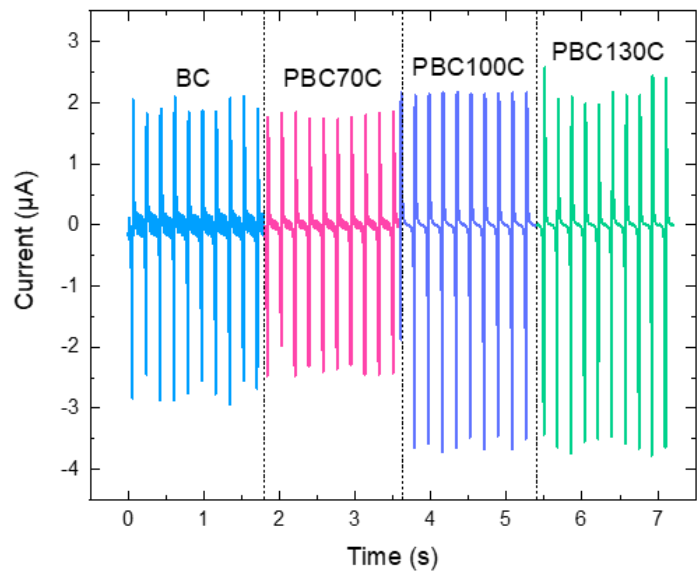

(d)

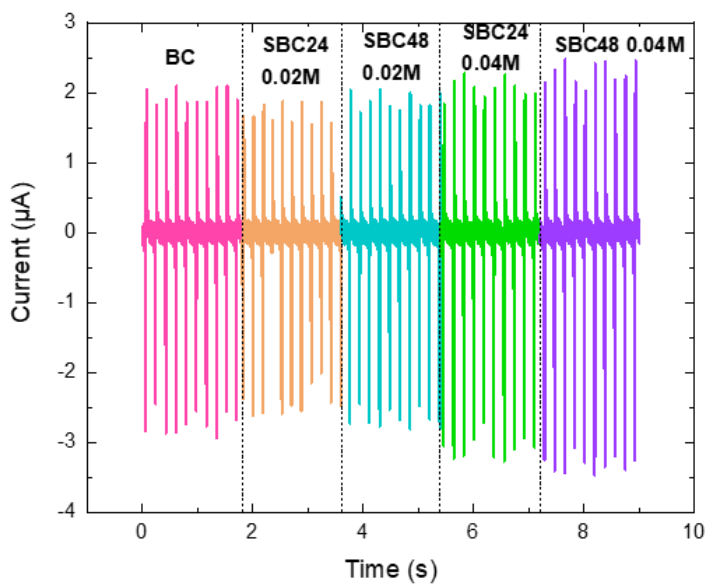

Figure 9. The TENG voltage and current output of $(a, b)$ the $B C$ and phosphorylated $B C(S B C)$ and $(c, d)$ the $B C$ and sulfonated $\mathrm{BC}(\mathrm{SBC})$.

The rougher surface means the better contact area between the pair of friction materials for enhancing charge transfer upon the pressing/releasing process [33]. Moreover, the denser film with fewer pores would result in better contact, which improves electrostatic induction and charge transfer [34]. As shown in the SEM images, in Fig. 7 and 8, the BC films are obviously denser after phosphorylation and sulfonation. Moreover, the BC surfaces became 
rougher as a result of phosphate and sulfonic groups' coating. Thus, the phosphorylated and sulfonated films, which were densely packed with more roughness, contribute to the enhanced TENG voltage and current output. The other cause could be attributed to the change in triboelectric potential of the BC surface after surface modification. In this work, the BC film was paired with PTFE, which is the most negatively charged in the triboelectric series [35]. When the BC surfaces were phosphorylated or sulfonated, the surface charges could be altered to become more positive than the unmodified $\mathrm{BC}[36,37]$. This factor also contributed to the enhanced voltage and current outputs.

\section{Conclusions}

In this work, we have successfully functionalized the $\mathrm{BC}$ film by phosphorylation and sulfonation processes. The characteristic functional groups on $\mathrm{BC}$ nanofibers were identified by using the FTIR analysis technique. The functionalization did not change the BC crystal structure nor the hydrophilic properties. However, it did change the surface morphology as well as the TENG output performance. The SEM images showed that phosphorylation and sulfonation resulted in more surface roughness as the functional groups coated on $\mathrm{BC}$ nanofibers. The $\mathrm{BC}$ fibers became more densely packed, and the pores between fibers were reduced. Consequently, when using the phosphorylated $\mathrm{BC}$ and the sulfonated $\mathrm{BC}$ pairing with PTFE in TENG test, the output performance (both voltage and current) was improved compared to the unmodified BC. The improved performance was believed to be due to the rougher and denser $\mathrm{BC}$ surface and the change in the $\mathrm{BC}$ triboelectric potential. Our work illustrated a simple way to improve the performance of the cellulose-based biocompatible TENG, which has potential use for harvesting environmental energy for self-powered implantable and wearable electronics.

\section{Funding}

This research was supported by (1) the Basic Research Fund of Khon Kaen University, and (2) the Research Network NANOTEC (RNN) program of the National Nanotechnology Center (NANOTEC), NSTDA, Ministry of Higher Education, Science, Research and Innovation, and Khon Kaen University, Thailand.

\section{Acknowledgments}

The authors declared no acknowledgments.

\section{Conflicts of Interest}

The authors declare no conflict of interest.

\section{References}

1. Song, Y.D.; Wang, N.; Wang, Y.H.; Zhang, R.Y.; Olin, H.; Yang, Y. Direct Current Triboelectric Nanogenerators. Adv. Energy Mater. 2020, 10, 2002756, https://doi.org/10.1002/aenm.202002756.

2. Kim, D.W., Lee, J.H., Kim, J.K., Jeong, U. Material aspects of triboelectric energy generation and sensors. NPG Asia Mater. 2020, 12, https://doi.org/10.1038/s41427-019-0176-0.

3. Khandelwal, G.; Raj, N.P.M.J.; Kim, S.J. Triboelectric nanogenerator for healthcare and biomedical applications. Nano Today 2020, 33, 100882, https://doi.org/10.1016/j.nantod.2020.100882. 
4. Zhou, Y.; Shen, M.; Cui, X.; Shao, Y.; Li, L.; Zhang, Y. Triboelectric nanogenerator based selfpowered sensor for artificial intelligence. Nano Energy 2021, 84, 105887, https://doi.org/10.1016/j.nanoen.2021.105887.

5. Kim, W.G.; Kim, D.W.; Tcho, I.W.; Kim, J.K.; Kim, M.S.; Choi, Y.K. Triboelectric Nanogenerator: Structure, Mechanism, and Applications. ACS Nano 2021, 15, 258-287, https://doi.org/10.1021/acsnano.0c09803.

6. Li, X.J.; Jiang, C.M.; Ying, Y.B.; Ping, J.F. Biotriboelectric Nanogenerators: Materials, Structures, and Applications. Adv. Energy Mater. 2020, 10, 2002001, https://doi.org/10.1002/aenm.202002001.

7. Li, W.; Pei, Y.; Zhang, C.; Kottapalli, A.G.P. Bioinspired designs and biomimetic applications of triboelectric nanogenerators. Nano Energy 2021, 84, 105865, https://doi.org/10.1016/j.nanoen.2021.105865.

8. He, W.; Fu, X.; Zhang, D.; zhang, Q.; Zhuo, K.; Yuan, Z.; Ma, R. Recent progress of flexible/wearable self-charging power units based on triboelectric nanogenerators. Nano Energy 2021, 84, 105880, https://doi.org/10.1016/j.nanoen.2021.105880.

9. Kim, H.J.; Kim, J.H.; Jun, K.W.; Kim, J.H.; Seung, W.C.; Kwon, O.H.; Park, J.Y.; Kim, S.W.; Oh, I.K. Silk Nanofiber-Networked Bio-Triboelectric Generator: Silk Bio-TEG. Adv. Energy Mater. 2016, 6, 1502329, https://doi.org/10.1002/aenm.201502329.

10. Jiang, W.; Li, H.; Liu, Z.; Li, Z.; Tian, J.J.; Shi, B.J.; Zou, Y.; Ouyang, H.; Zhao, C.C.; Zhao, L.M.; Sun, R.; Zheng, H.R.; Fan, Y.B.; Wang, Z.L.; Li, Z. Fully Bioabsorbable Natural-Materials-Based Triboelectric Nanogenerators. Adv. Mater. 2018, 30, 1801895, https://doi.org/10.1002/adma.201801895.

11. Wang, R.X.; Gao, S.J.; Yang, Z.; Li, Y.L.; Chen, W.N.; Wu, B.X.; Wu, W. Engineered and LaserProcessed Chitosan Biopolymers for Sustainable and Biodegradable Triboelectric Power Generation. Adv. Mater. 2018, 30, 1706267, https://doi.org/10.1002/adma.201706267.

12. Ccorahua, R.; Huaroto, J.; Luyo, C.; Quintana, M.; Vela, E.A. Enhanced-performance biotriboelectric nanogenerator based on starch polymer electrolyte obtained by a cleanroom-free processing method. Nano Energy 2019, 59, 610-618, https://doi.org/10.1016/j.nanoen.2019.03.018.

13. Torres, F.G.; De-la-Torre, G.E. Polysaccharide-based triboelectric nanogenerators: A review. Carbohydr. Polym. 2021, 251, https://doi.org/10.1016/j.carbpol.2020.117055.

14. Chen, Y.M.; Zhang, L.; Yang, Y.; Pang, B.; Xu, W.H.; Duan, G.G.; Jiang, S.H.; Zhang, K. Recent Progress on Nanocellulose Aerogels: Preparation, Modification, Composite Fabrication, Applications. Adv. Mater. 2021, 33, 2005569, https://doi.org/10.1002/adma.202005569.

15. Ahankari, S.S.; Subhedar, A.R.; Bhadauria, S.S.; Dufresne, A. Nanocellulose in food packaging: A review. Carbohydr. Polym. 2021, 255, https://doi.org/10.1016/j.carbpol.2020.117479.

16. Yao, C.H.; Hernandez, A.; Yu, Y.H.; Cai, Z.Y.; Wang, X.D. Triboelectric nanogenerators and power-boards from cellulose nanofibrils and recycled materials. Nano Energy 2016, 30, 103-108, https://doi.org/10.1016/j.nanoen.2016.09.036.

17. He, X.; Zou, H.Y.; Geng, Z.S.; Wang, X.F.; Ding, W.B.; Hu, F.; Zi, Y.L.; Xu, C.; Zhang, S.L.; Yu, H.; Xu, M.Y.; Zhang, W.; Lu, C.H.; Wang, Z.L. A Hierarchically Nanostructured Cellulose FiberBased Triboelectric Nanogenerator for Self-Powered Healthcare Products. Adv. Funct. Mater. 2018, 28, 1805540, https://doi.org/10.1002/adfm.201805540.

18. Zheng, Q.F.; Fang, L.M.; Guo, H.Q.; Yang, K.F.; Cai, Z.Y.; Meador, M.A.B.; Gong, S.Q. Highly Porous Polymer Aerogel Film-Based Triboelectric Nanogenerators. Adv. Funct. Mater. 2018, 28, 1706365, https://doi.org/10.1002/adfm.201706365.

19. Eslahi, N.; Mahmoodi, A.; Mahmoudi, N.; Zandi, N.; Simchi, A. Processing and Properties of Nanofibrous Bacterial Cellulose-Containing Polymer Composites: A Review of Recent Advances for Biomedical Applications. Polym. Rev. 2020, 60, 144-170, https://doi.org/10.1080/15583724.2019.1663210. 
20. Parte, F.G.B.; Santoso, S.P.; Chou, C.C.; Verma, V.; Wang, H.T.; Ismadji, S.; Cheng, K.C. Current progress on the production, modification, and applications of bacterial cellulose. Crit. Rev. Biotechnol. 2020, 40, 397-414, https://doi.org/10.1080/07388551.2020.1713721.

21. Sriplai, N.; Pinitsoontorn, S. Bacterial cellulose-based magnetic nanocomposites: A review. Carbohydr. Polym. 2021, 254, 117228, https://doi.org/10.1016/j.carbpol.2020.117228.

22. Kim, H.J.; Yim, E.C.; Kim, J.H.; Kim, S.J.; Park, J.Y.; Oh, I.K. Bacterial Nano-Cellulose Triboelectric Nanogenerator. Nano Energy 2017, 33, 130-137, https://doi.org/10.1016/j.nanoen.2017.01.035.

23. Shao, Y.; Feng, C.P.; Deng, B.W.; Yin, B.; Yang, M.B. Facile method to enhance output performance of bacterial cellulose nanofiber based triboelectric nanogenerator by controlling micro-nano structure and dielectric constant. Nano Energy 2019, 62, 620-627, https://doi.org/10.1016/j.nanoen.2019.05.078.

24. Jakmuangpak, S.; Prada, T.; Mongkolthanaruk, W.; Harnchana, V.; Pinitsoontorn, S. Engineering Bacterial Cellulose Films by Nanocomposite Approach and Surface Modification for Biocompatible Triboelectric Nanogenerator. ACS Appl. Electron. Mater. 2020, 2, 2498-2506, https://doi.org/10.1021/acsaelm.0c00421.

25. Yao, C.H.; Yin, X.; Yu, Y.H.; Cai, Z.Y.; Wang, X.D. Chemically Functionalized Natural Cellulose Materials for Effective Triboelectric Nanogenerator Development. Adv. Funct. Mater. 2017, 27, 1700794, https://doi.org/10.1002/adfm.201700794.

26. Thomas, B.; Raj, M.C.; Athira, K.B.; Rubiyah, M.H.; Joy, J.; Moores, A.; Drisko, G.L.; Sanchez, C. Nanocellulose, a Versatile Green Platform: From Biosources to Materials and Their Applications. Chem Rev 2018, 118, 11575-11625, https://doi.org/10.1021/acs.chemrev.7b00627.

27. Kokol, V.; Bozic, M.; Vogrincic, R.; Mathew, A.P. Characterisation and properties of homo- and heterogenously phosphorylated nanocellulose. Carbohydr. Polym. 2015, 125, 301-313, https://doi.org/10.1016/j.carbpol.2015.02.056.

28. Yue, L.N.; Xie, Y.J.; Zheng, Y.D.; He, W.; Guo, S.L.; Sun, Y.; Zhang, T.; Liu, S.M. Sulfonated bacterial cellulose/polyaniline composite membrane for use as gel polymer electrolyte. Compos Sci Technol 2017, 145, 122-131, https://doi.org/10.1016/j.compscitech.2017.04.002.

29. Sintusiri, J.; Harnchana, V.; Amornkitbamrung, V.; Wongsa, A.; Chindaprasirt, P. Portland Cement-TiO2 triboelectric nanogenerator for robust large-scale mechanical energy harvesting and instantaneous motion sensor applications. Nano Energy 2020, 74, 104802, https://doi.org/10.1016/j.nanoen.2020.104802.

30. Chanthiwong, M.; Mongkolthanaruk, W.; Eichhorn, S.J.; Pinitsoontorn, S. Controlling the processing of co-precipitated magnetic bacterial cellulose/iron oxide nanocomposites. Mater. Design 2020, 196, 109148, https://doi.org/10.1016/j.matdes.2020.109148.

31. Sriplai, N.; Mongkolthanaruk, W.; Eichhorn, S.J.; Pinitsoontorn, S. Magnetic bacterial cellulose and carbon nanofiber aerogel by simple immersion and pyrolysis. J. Mater. Sci. 2020, 55, $4113-$ 4126, https://doi.org/10.1007/s10853-019-04295-w.

32. Pacaphol, K.; Aht-Ong, D. The influences of silanes on interfacial adhesion and surface properties of nanocellulose film coating on glass and aluminum substrates. Surf. Coat. Technol. 2017, 320, 70-81, https://doi.org/10.1016/j.surfcoat.2017.01.111.

33. Singh, H.H.; Khare, N. Improved performance of ferroelectric nanocomposite flexible film based triboelectric nanogenerator by controlling surface morphology, polarizability, and hydrophobicity. Energy 2019, 178, 765-771, https://doi.org/10.1016/j.energy.2019.04.150.

34. Wu, C.S.; Wang, A.C.; Ding, W.B.; Guo, H.Y.; Wang, Z.L. Triboelectric Nanogenerator: A Foundation of the Energy for the New Era. Adv. Energy Mater. 2019, 9, https://doi.org/10.1002/aenm.201802906.

35. Kim, Y.J.; Lee, J.; Park, S.; Park, C.; Park, C.; Choi, H.J. Effect of the relative permittivity of oxides on the performance of triboelectric nanogenerators. RSC Adv. 2017, 7, 49368-49373, https://doi.org/10.1039/c7ra07274k. 
36. Blilid, S.; Kedzierska, M.; Milowska, K.; Wronska, N.; El Achaby, M.; Katir, N.; Belamie, E.; Alonso, B.; Lisowska, K.; Lahcini, M.; Bryszewska, M.; El Kadib, A. Phosphorylated Micro- and Nanocellulose-Filled Chitosan Nanocomposites as Fully Sustainable, Biologically Active Bioplastics. ACS Sustain. Chem. Eng. 2020, 8, 18354-18365, https://doi.org/10.1021/acssuschemeng.0c04426.

37. Wang, Y.X.; Lwal, A.L.J.; Wang, Q.; Zhou, J.; Dufresne, A.; Lin, N. Regulating surface sulfonation on cellulose nanocrystals and self-assembly behaviors. Chem. Commun. 2020, 56, 10958-10961, https://doi.org/10.1039/d0cc04256k. 